Seamless Integration of DM with DBMS \title{
and Applications
}

\section{Hongjun Lu}

Hong Kong University of Science \& Technology

Email:luhj@cs.ust.hk

URL: http://www.cs.ust.hk/ luhj 


\section{Main Arguments}

It is a critical issue

to sustain KDD research.

to make DM a main stream technology

It is not really a new problem, but worth more attention from researchers

It should be addressed from various directions 


\section{A Very Hot Research Topic Could Die}

Deductive database

Excellent topic/work

Little application support

- Absorbed by RDBMS Extensions in RDBMS

\section{OODB}

Good piece of work

There are application support

Did not become main stream technology

- Absorbed by RDBMS OR-DBMS

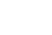




\section{What Makes KDD Different?}

Experience of RDBMS

Turn theory a popular technology

What will really distinguish KDD from other dada analysis research work

Scalability?

Making sophisticate/theoretical work a main stream technology on the market! 


\section{Making DM a Main Stream Technology}

Data is everywhere, database is every where, why not data mining?

Unfortunately, not yet

DM has been then for a decade, not a main stream technology yet

Perceived to be sophisticated technology, usable only by specialists

Long, expensive projects

Stand-alone, loosely-coupled with data infrastructures

Difficult to infuse into existing mission-critical applications

-- R. Agrawal, KDD'99 


\section{Efforts Have Been Made}

Has been an open problem

Emphasized in tutorials, speeches

Various efforts have been made

Vendors:

- both DM software vendors/ DBMS vendors

Researchers:

- DBMS side: functionality/language extensions

- DM side: DBMS/SQL-aware mining

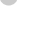

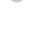




\section{Association Rule Mining and DBMS}

Houtsma and Swami, ICDE95

SQL implementation of Apriori-based association rule mining

Agrawal and Shim, KDD96

Tight coupling, UDF aproach

Sarawagi, Thomas and Agrawal, SIGMOD98

Association rule mining based on SQL-92 and ORDBMS

Thomas and Sarawagi, KDD98

Generalized AR/sequential mining using SQL queries

Thomas and Chakravarthy, DaWak99

Performance evaluation of SQL-92 based AR mining

Pramudiono, Shintani, Tamura and Kitsuregawa, PAKDD99, Yoshizawa, Pramudiono, and Kitsuregawa, DaWak00

Parallel mining of generalized AR using SQL 


\section{SQL-Aware Classification}

Wang, Iyer, and Vitter, IDEAL98

Decision tree based algorithm

Using UDF to implement required computations

Chaduri, Fayyad \& Bernhardt, ICDE'99

Middleware between SQL Server \& classification

algorithms

Emphasizing performance

$\mathrm{Lu}$ and Liu, $V L D B 2000$

Scalable classifier

Implemented using DB2 SQL statements 


\section{How about Performance}

Yoshizawa, Pramudiono, Kitsuregawa DaWak'00 IBM RS6000 SP (12 nodes)

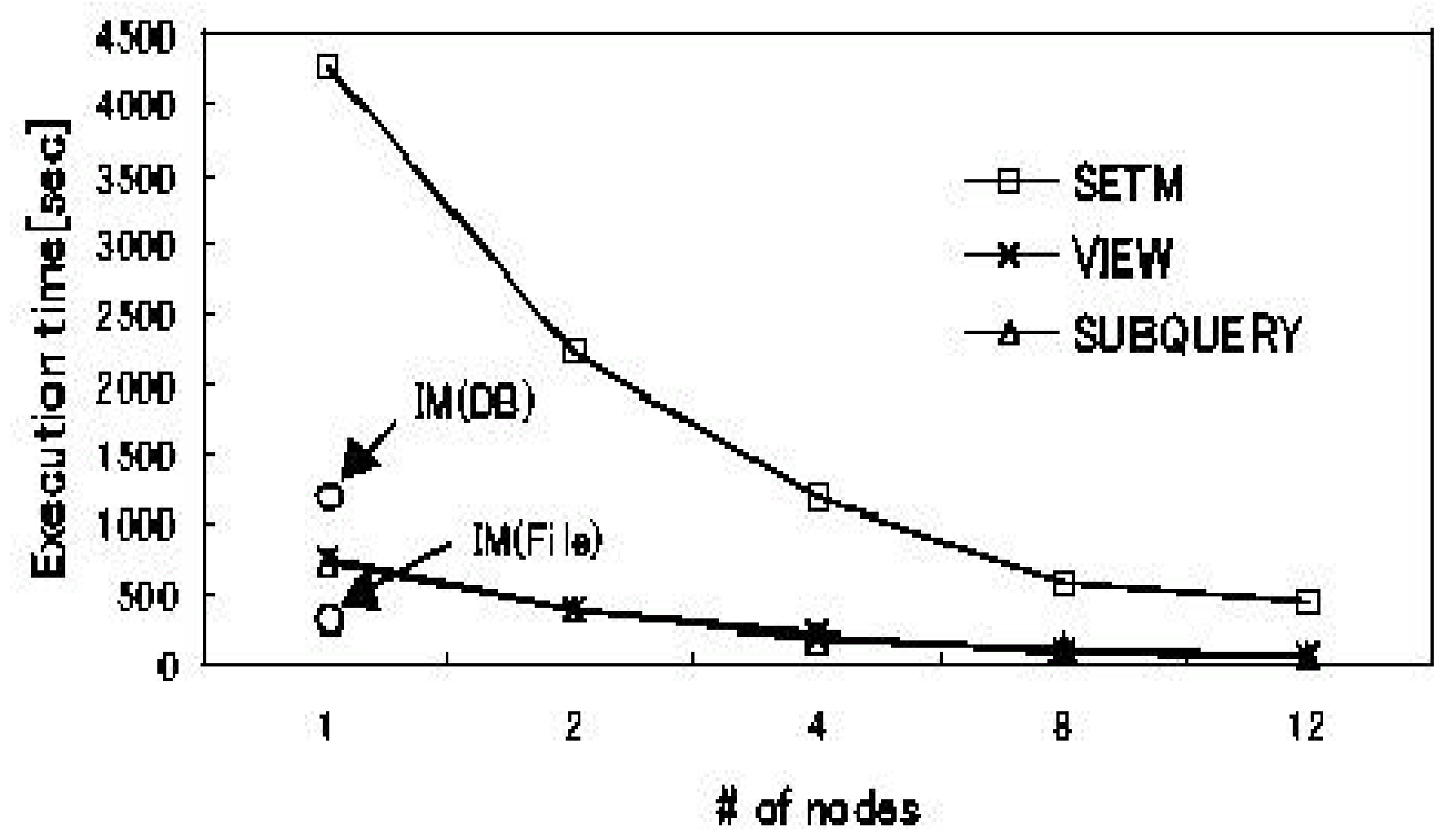




\section{Compare with SLIQ (Function 5)}

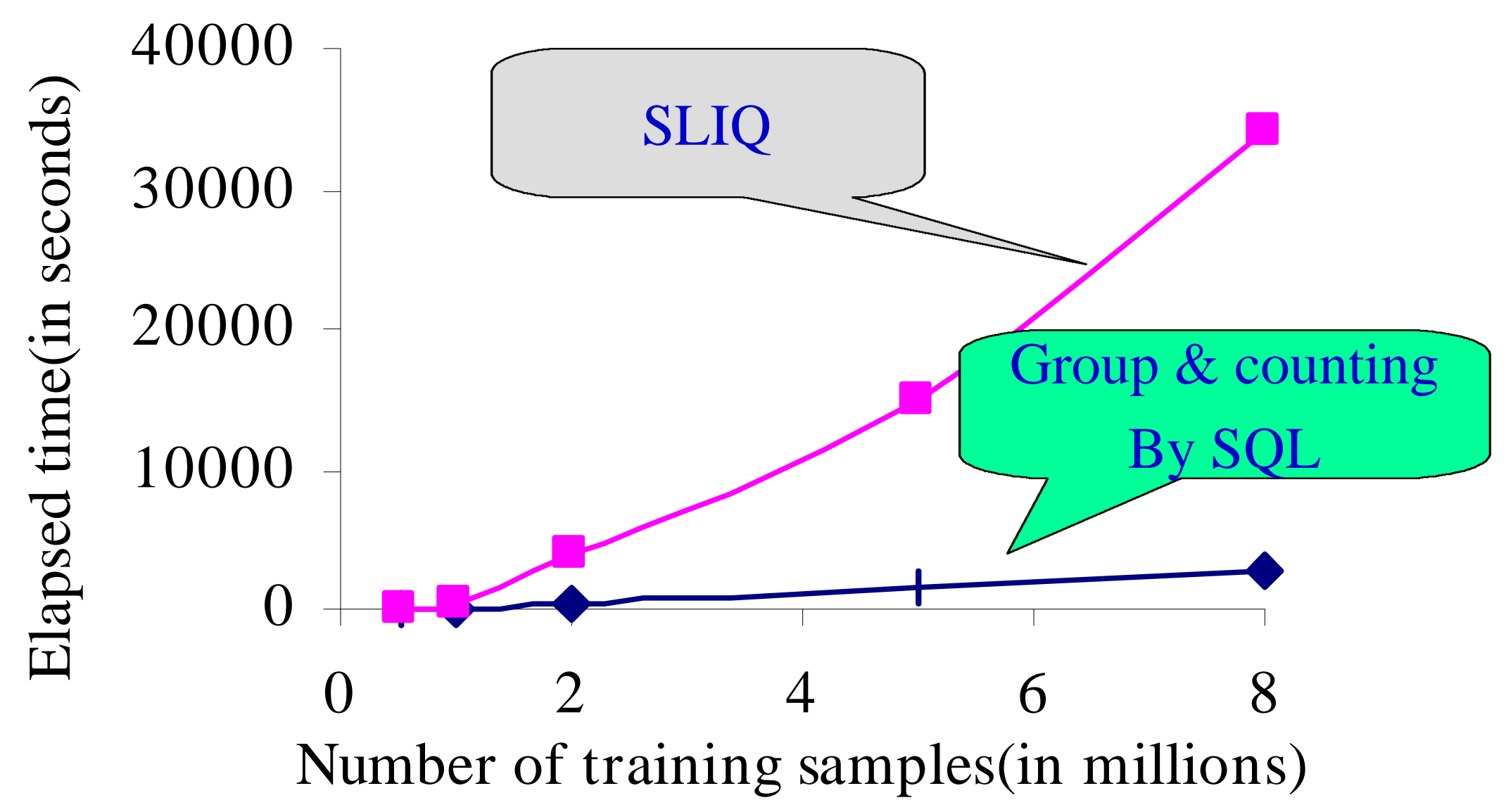

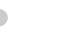

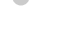

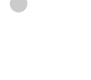




\section{Compare with CBA (Function 10)}

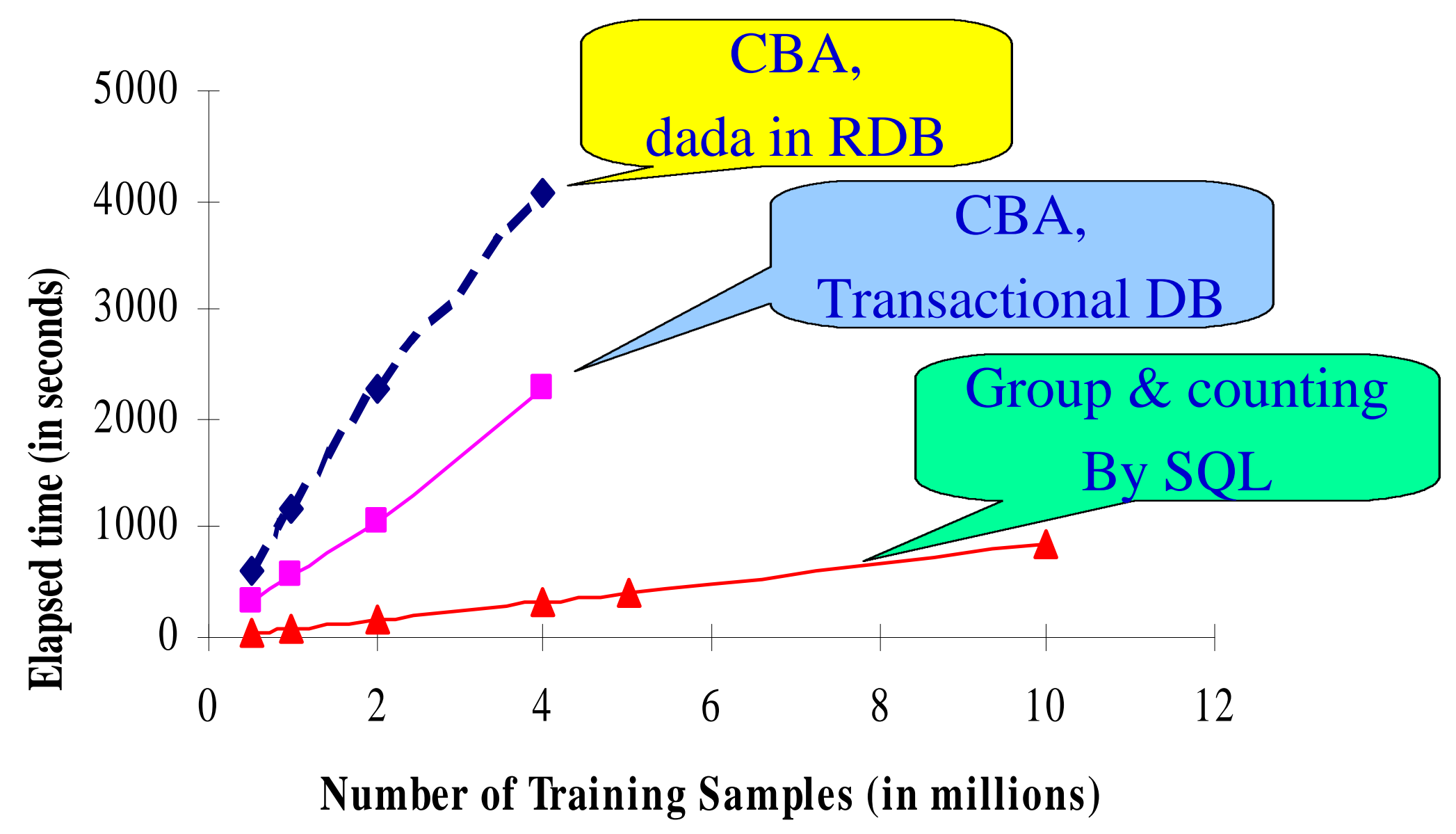




\section{Seamless Integration}

A wide range of approaches Integration of DM \& DBMS

ODBC data access

Elegant data staging/caching

Stored procedures and user defined functions

DM as DBMS applications

Integration DM \& applications

Uniform/standard interface

Application-friendliness data mining

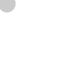




\section{Integration of DM and DBMS}

machine learning/statistical algorithms

main memory based (e.g. C4.5)

disk aware algorithms

data on the disk, I/O cost should be minimized (Agrawl'92)

DBMS aware algorithms

tight/smart control of interaction between DM algorithms and DBMS (e.g. Chaduri, Fayyad \& Bernhardt, ICDE'99)

SQL aware algorithms

employ stored procedures/UDF (e.g.Wang, Iyer, and Vitter, IDEAL98)

DM implemented as embedded SQL programs

employ DBMS functions only (e.g. Lu \& Liu, VLDB00) 


\section{Our Experience -- Classification}

Starting with efficient classification

one of most popular DM problems

Using RDBMS native functions

push as much data processing to RDBMS as possible

- Performance

- Scalability

- Leverage

Model (the classifier built) as first class citizen (table) of database

facilitate integration with applications 


\section{Classification - Decision Trees}

Various algorithms have been developed

Decision tree: one of the popular methods

\begin{tabular}{|c|c|c|}
\hline age-group & car-type & risk \\
\hline young & family & high \\
\hline young & sport & high \\
\hline middle & sport & high \\
\hline old & family & low \\
\hline middle & family & low \\
\hline
\end{tabular}

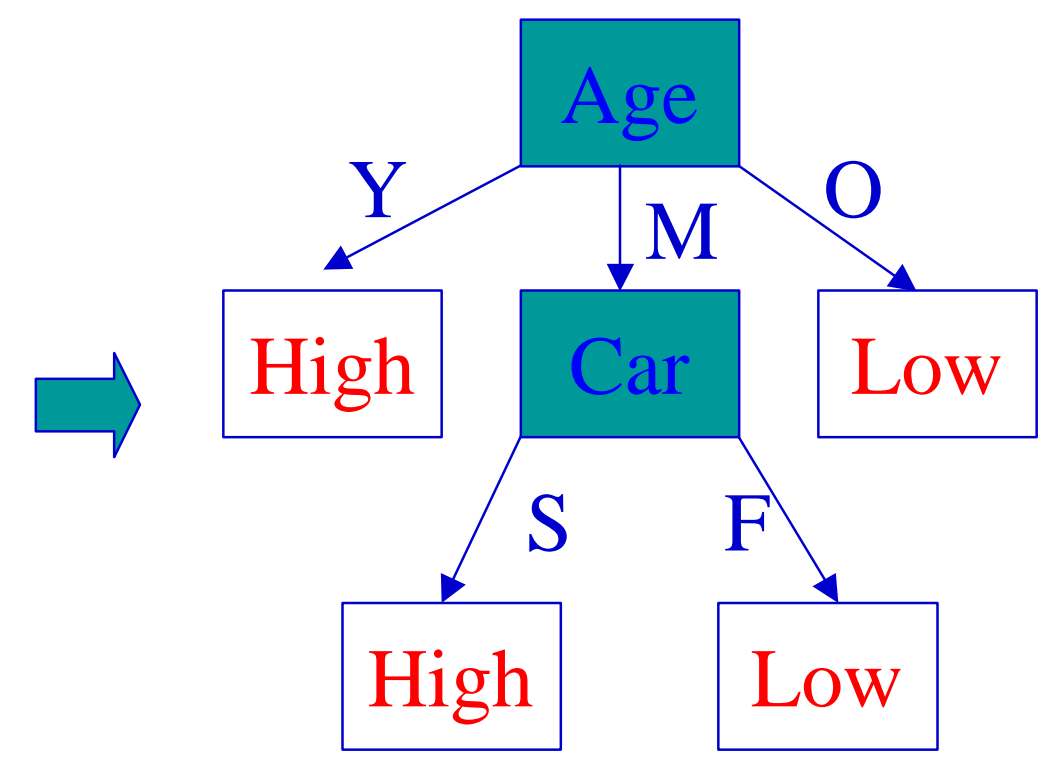




\section{Classifier as Relational Tables}

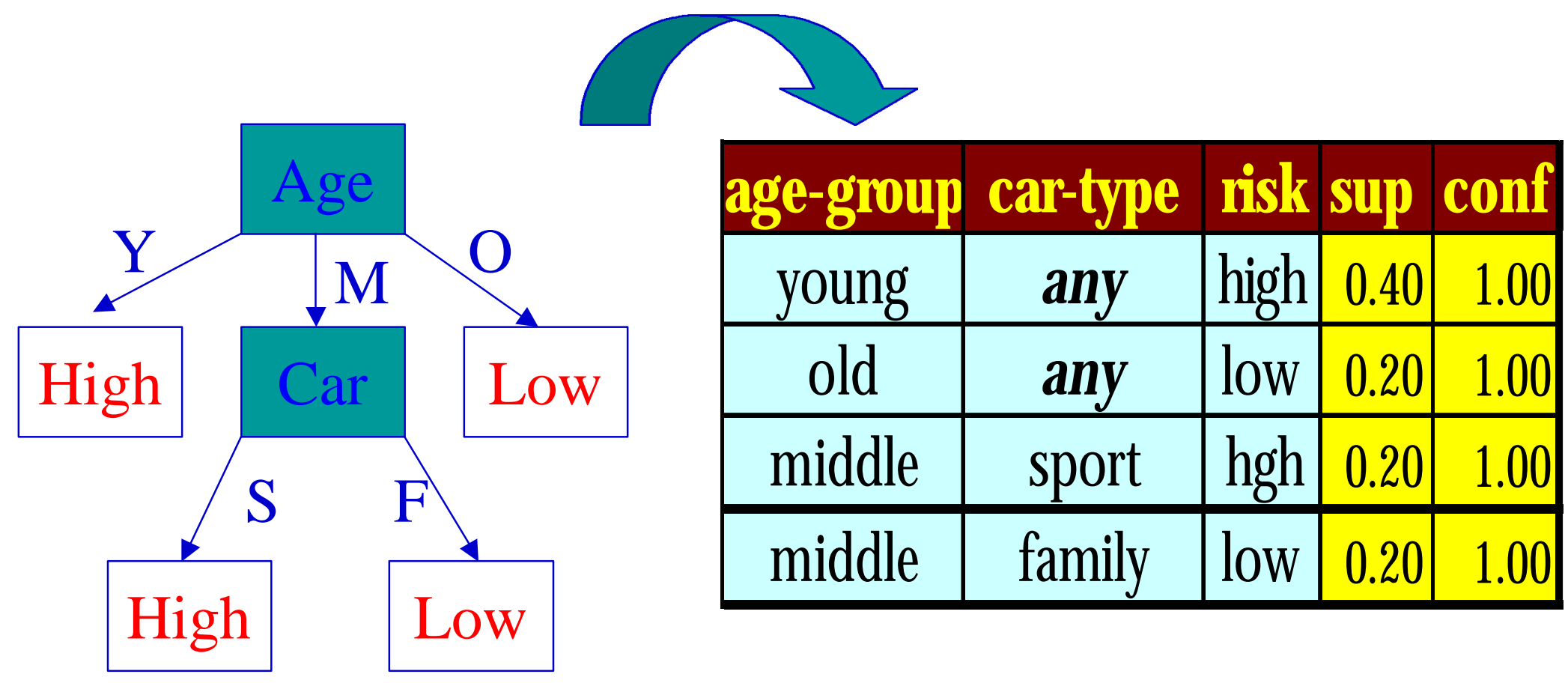




\section{Using the Classifier: Case One}

Given tuple $u\left(a_{1 u}, a_{2 u}, \ldots, a_{n u}\right)$, one matching row $\mathrm{r}_{\mathrm{i}}\left(\mathrm{a}_{\mathrm{ii}}, \mathrm{a}_{2 \mathrm{i}}, \ldots, \mathrm{a}_{\mathrm{ii}}, \mathrm{c}\right.$, sup, conf $)$ found Class of $u$ is $\mathbf{c}_{\mathbf{i}}$.

(young, family)?

$$
\text { risk }=\text { high }
$$

$\longrightarrow$\begin{tabular}{|c|c|c|c|c|}
\hline age-group & car-type & risk & sup & conf \\
\hline young & any & high & 0.4 & 1.00 \\
\hline old & any & low & 0.2 & 1.00 \\
\hline any & sport & high & 0.4 & 1.00 \\
\hline middle & family & low & 0.2 & 1.00 \\
\hline
\end{tabular}

$$
\text { risk }=\text { low }
$$




\section{Using the Classifier: Case One}

Given tuple $u\left(a_{1 u}, a_{2 u}, \ldots, a_{n u}\right)$, more than one matching row is found:

select the one with highest confidence

If there are ties, the class with highest support will be assigned to $\mathrm{C}_{\mathrm{u}}$.

If there are still ties, one randomly picked from them will be assigned to $\mathrm{c}$.

(old, sport) ?

\begin{tabular}{|c|c|c|c|c|}
\hline age-group & car-type & risk & sup & conf \\
\hline young & any & high & 0.4 & 1.00 \\
\hline old & any & low & 0.2 & 1.00 \\
\hline any & sport & high & 0.4 & 1.00 \\
\hline middle & family & low & 0.2 & 1.00 \\
\hline
\end{tabular}




\section{Using the Classifier: Case Three}

Given tuple $u\left(a_{1 u}, a_{2 u}, \ldots, a_{n u}\right)$, no matching row is found Compute $\mathrm{P}\left(\mathrm{C}_{\mathrm{k}} \mid \mathrm{u}\right)$ based on Bayes theorem

$P\left(c_{k} \mid u\right) \infty p\left(c_{k}\right) \prod_{i=1}^{n} p\left(a_{i u} \mid c_{k}\right)=\frac{\prod_{i=1}^{n} p\left(a_{i u} \wedge c_{k}\right)}{p\left(c_{k}\right)^{n-1}}$

Class $\mathrm{C}_{\mathrm{k}}$ with the highest probability is assigned to $\mathrm{u}$ 


\begin{tabular}{|c|c|c|}
\hline age-group & car-type & risk \\
\hline young & family & high \\
\hline young & sport & high \\
\hline middle & sport & high \\
\hline old & family & low \\
\hline middle & family & low \\
\hline
\end{tabular}

$\begin{aligned} & \text { \# occurrences of samples with } \\
& \text { the same attribute values }\end{aligned}$
\begin{tabular}{|c|c|c|c|}
\hline age-group & car-type & risk & count \\
\hline young & any & high & 2 \\
\hline middle & any & high & 1 \\
\hline middle & any & low & 1 \\
\hline old & any & low & 1 \\
\hline any & family & high & 1 \\
\hline any & family & low & 2 \\
\hline any & sport & high & 2 \\
\hline young & family & high & 1 \\
\hline young & sport & high & 1 \\
\hline middle & sport & high & 1 \\
\hline middle & family & low & 1 \\
\hline old & family & low & 1 \\
\hline
\end{tabular}




\begin{tabular}{|c|c|c|c|c|c|c|c|c|c|}
\hline age-group & car-type & risk & count & conf & & & & & \\
\hline young & any & high & 2 & 1.00 & & & & & \\
\hline middle & any & high & 1 & -0.50 & & & & & \\
\hline middule- & âfy & low & 1 & 0.50 & Low con & fidence & & & \\
\hline old & any & low & 1 & 1.00 & & & & & \\
\hline$a n y$ & family & high & 1 & 0.33 & age-group & car-type & risk & sup & conf \\
\hline any & family & low & 2 & 0.67 & young & any & high & 0.4 & 1.00 \\
\hline any & sport & high & 2 & 1.00 & old & any & low & 0.2 & 1.00 \\
\hline young & family & hight & 1 & 1.00 & ANY & sport & high & 0.4 & 1.00 \\
\hline youning & spoit & high & 1 & 1.00 & middle & family & low & 0.2 & 1.00 \\
\hline middle & sport & hight & 1 & 1.00 & \multirow{3}{*}{\multicolumn{4}{|c|}{ Redundant }} & \\
\hline middle & family & low & 1 & 1.00 & & & & & \\
\hline old & family & low & 1 & 1.00 & & & & & \\
\hline
\end{tabular}




\section{Example of grouping sets query}

SELECT age-group, car-type, risk, count(*) as count

FROM insurance GROUP BY GROUPING SETS (age-group, car-type), risk

\begin{tabular}{|c|c|c|}
\hline \multicolumn{3}{l}{} \\
\hline age-group car-type & risk \\
\hline young & family & high \\
\hline young & sport & high \\
\hline middle & sport & high \\
\hline old & family & low \\
\hline middle & family & low \\
\hline
\end{tabular}

\begin{tabular}{|c|c|c|c|}
\hline age-group car-type & risk & count \\
\hline middle & - & high & 1 \\
\hline middle & - & low & 1 \\
\hline old & - & low & 1 \\
\hline young & - & high & 2 \\
\hline- & family & high & 1 \\
\hline- & family & low & 2 \\
\hline- & sport & high & 2 \\
\hline
\end{tabular}




\section{Computing Class Populations}

SELECT $A_{1}, A_{2}, . ., A_{n}$ class, count $(*)$ FROM TrainD

GROUP BY $A_{k}$, CUBE $\left(A_{1}, A_{2}, .\right.$. $\left.A_{k-1}, A_{k+1} . ., A_{n}\right)$, class

ORDER BY $A_{1}, A_{2}, . ., A_{n}$

where is $A_{k}$ the best splitting attribute.

\begin{tabular}{|c|c|c|}
\hline age-group & car-type & risk \\
\hline young & family & high \\
\hline young & sport & high \\
\hline middle & sport & high \\
\hline old & family & low \\
\hline middle & family & low \\
\hline
\end{tabular}

SELECT age-group, car-type, risk,count $(*)$ FROM insurance

GROUP BY agegrap CUBE (car-type), risk

\begin{tabular}{|c|c|c|c|}
\hline age-group & car-type & risk & count \\
\hline middle & any & high & 1 \\
\hline middle & any & low & 1 \\
\hline old & any & low & 1 \\
\hline young & any & high & 2 \\
\hline middle & sport & high & 1 \\
\hline middle & family & low & 1 \\
\hline old & family & low & 1 \\
\hline young & family & high & 1 \\
\hline young & sport & high & 1 \\
\hline
\end{tabular}




\section{Classification Meets DBMS}

The most demanding computation in classification

Class population

- entropy, gini index, contingency tables,

No more than computing the data

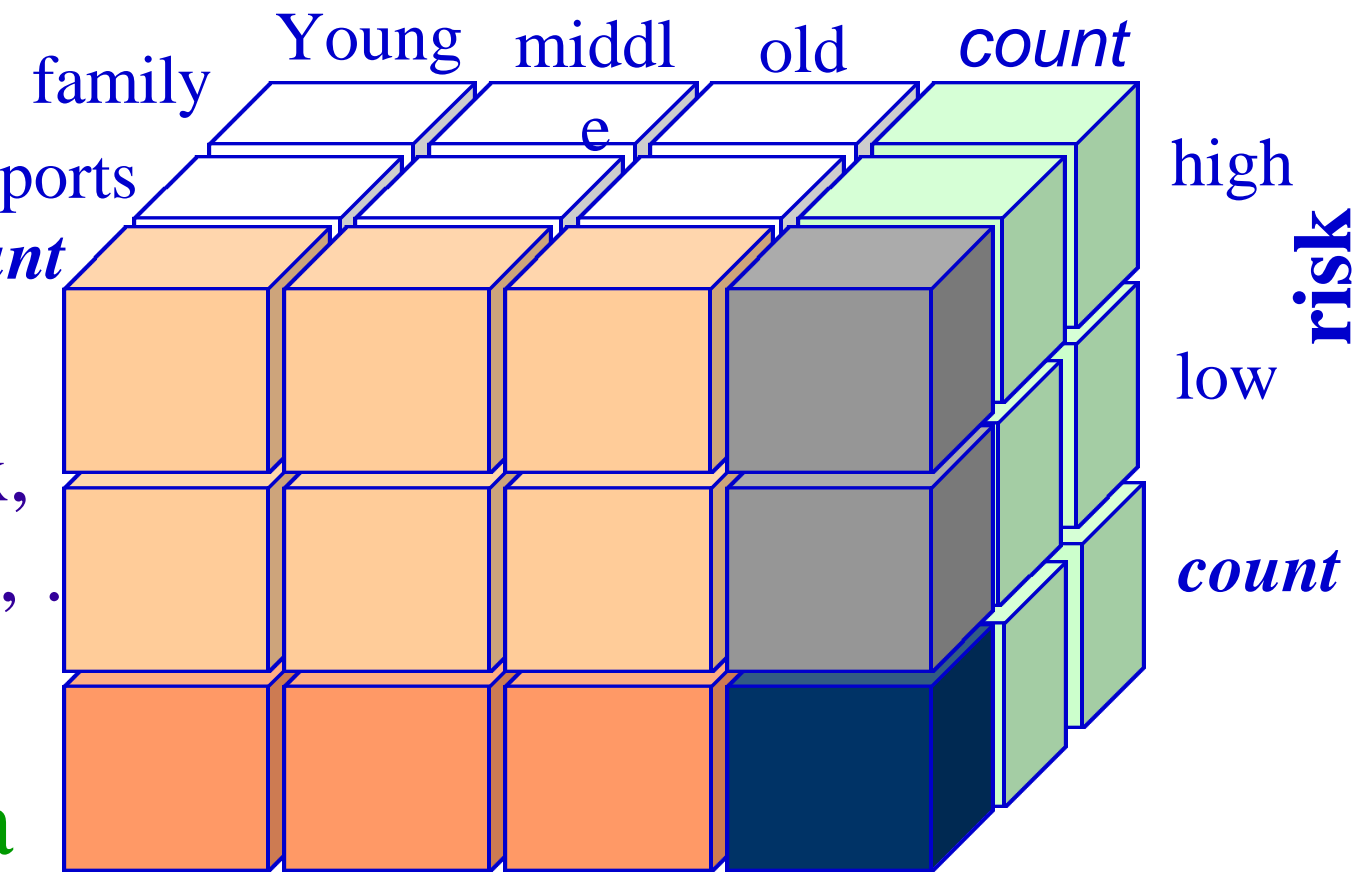
cube! 


\section{Implementation Issues/Solutions}

Data cube computation is expensive in DBMS

Large \# of attributes High dimension

Large \# of distinct attribute values large \# of groups

Optimization techniques addressing the issue

Reducing dimension by one

- selecting a starting attribute

Generating the classifier in iterations:

- each iteration computes a sub-cubes and generates rules involving selected attributes. 


\section{What Learned}

A SQL based classification algorithm can be developed fully implemented as an embedded SQL program without UDF

Classifier obtained is a relational table

Performance seems not an issue

Better classification accuracy

Execution speed \& scalability issue solvable.

How about other DM problems?

-

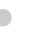

- 


\section{Integration of DM and Application}

Standard interfaces, specifications for

Input data

Mining parameter

Output of mining (the model)

Sample efforts

OLE-DB for data mining

PMML (Predictive Model Markup Language)

- an XML-based to define predictive models for sharing 


\section{The Real Challenge}

The real challenge faced by users of DM technology/applications

What to mine?

What technique to use?

Given technique, what method/algorithm to use

With chosen method/algorithm, how to set parameters 


\section{Application Friendly Mining Algorithms}

Algorithm selection

Parameter reduction

Parameter selection/transformation 


\section{Algorithm Selection}

Auto, semi-auto algorithm selection?

Impossible?

- Set a modest goal

- How about allow you to scan data once/sample data before making decision

Application dependency?

- vertical market

- Turn-key systems

- $\quad$ inadequate research in integrating domain knowledge!

Benchmarking/analyzing existing algorithms

Bench marking data 


\begin{tabular}{|c|c|c|c|c|c|c|c|c|c|}
\hline \multirow{2}{*}{ Data set } & \multicolumn{4}{|c|}{ Properties } & \multicolumn{5}{|c|}{ Accuracy } \\
\hline & \#attrs & \#classes & \# train & \# test & \begin{tabular}{|l|l|} 
C4.5 & NB \\
\end{tabular} & TAN & CBA & LB & GAC \\
\hline Australian & 14 & 2 & 690 & CV-10 & \begin{tabular}{|l|l|}
0.8437 \\
\end{tabular} & 0.852 & 0.855 & 0.857 & 0.883 \\
\hline Chess & 36 & 2 & 2,130 & 1,065 & \begin{tabular}{|l|l|}
0.995 & 0.872 \\
\end{tabular} & 0.921 & 0.981 & 0.902 & 0.944 \\
\hline Diabetes & 8 & 2 & 768 & CV-10 & \begin{tabular}{|l|l|}
0.717 & 0.751 \\
\end{tabular} & 0.765 & 0.729 & 0.767 & 0.767 \\
\hline Flare & 10 & 2 & 1,066 & CV-10 & \begin{tabular}{|l|l|}
0.812 & 0.795 \\
\end{tabular} & 0.826 & 0.831 & 0.815 & 0.843 \\
\hline German & 20 & 2 & 1,000 & CV-10 & \begin{tabular}{|l|l|}
0.717 & 0.741 \\
\end{tabular} & 0.727 & 0.732 & 0.748 & 0.768 \\
\hline Heart & 13 & 2 & 270 & CV-10 & \begin{tabular}{|l|l|}
0.767 & 0.822 \\
\end{tabular} & 0.833 & 0.819 & 0.822 & 0.838 \\
\hline Letter & 16 & 26 & 15,000 & 500 & \begin{tabular}{|l|l|}
0.777 & 0.749 \\
\end{tabular} & 0.857 & 0.518 & 0.764 & 0.800 \\
\hline Lymph & 18 & 4 & 148 & CV-10 & \begin{tabular}{|l|l|}
0.784 & 0.819 \\
\end{tabular} & 0.838 & 0.773 & 0.846 & 0.839 \\
\hline Pima & 8 & 2 & 768 & CV-10 & \begin{tabular}{|l|l|}
0.711 & 0.759 \\
\end{tabular} & 0.758 & 0.730 & 0.758 & 0.780 \\
\hline Satima & 36 & 6 & 4,435 & 2,000 & \begin{tabular}{|l|l|}
0.852 & 0.818 \\
\end{tabular} & 0.872 & 0.849 & 0.839 & $\mid 0.847$ \\
\hline$\overline{\text { Segme }}$ & 19 & 7 & 1,540 & 770 & \begin{tabular}{|l|l|}
0.958 & 0.918 \\
\end{tabular} & 0.935 & 0.935 & 0.942 & 0.943 \\
\hline Splice & 60 & 3 & 2,126 & 1,064 & \begin{tabular}{|l|l|}
0.933 & 0.946 \\
\end{tabular} & 0.946 & 0.700 & 0.946 & 0.956 \\
\hline Shuttle- & 9 & 7 & 38,661 & 934 & \begin{tabular}{|l|l|}
0.995 & 0.987 \\
\end{tabular} & 0.996 & 0.995 & \begin{tabular}{|l|}
0.994 \\
\end{tabular} & 0.998 \\
\hline Vehicle & 18 & 4 & 846 & $\mathrm{CV}-10$ & \begin{tabular}{|c|c|}
0.698 & 0.611 \\
\end{tabular} & 0.709 & 0.688 & \begin{tabular}{|l|}
0.688 \\
\end{tabular} & 0.681 \\
\hline Voting & 16 & 2 & 435 & CV-10 & \begin{tabular}{|l|l|}
0.957 & 0.903 \\
\end{tabular} & 0.933 & 0.935 & 0.947 & 0.956 \\
\hline Wavefo & 21 & 3 & 300 & 4,700 & \begin{tabular}{|l|l|l|}
0.704 & 0.785 \\
\end{tabular} & 0.791 & 0.753 & 0.794 & 0.761 \\
\hline Y east & 8 & 10 & 1,484 & CV-10 & \begin{tabular}{|l|l|}
0.557 & 0.581 \\
\end{tabular} & 0.572 & 0.551 & 0.582 & 0.574 \\
\hline$\overline{\text { AVERAGE }}$ & & & & & \begin{tabular}{|l|l|}
0.810 & 0.807 \\
\end{tabular} & 0.831 & 0.787 & 0.824 & 0.834 \\
\hline
\end{tabular}




\section{Parameter Reduction}

Eliminating as much parameters as possible

Why do we have parameters in algorithms at the first place?

Control the execution

Tuning the performance

Convenience for algorithm design

What kind of parameters can be removed? 


\section{Parameter Transformation}

Algorithm-oriented versus application oriented

Providing the mapping between application parameters and algorithm parameters 


\section{Conclusions}

Seamless integration is a critical issue

The problem can be addressed from different angles

Defining standard interface for data/model exchange

Developing DBMS/SQL based mining algorithms

Making mining algorithms application friendly

The problems are not easy to solve, not too many people are willing to solve, and that is why we, academic researchers, exist!

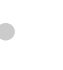

